\title{
Corrigendum: Partial second toe pulp free flaps in early childhood
}

Min Ki Hong, Dong Chul Lee, Min Suk Choi, Sung Hoon Koh, Jin Soo Kim, Si Young Roh, Kyung Jin Lee

Department of Plastic and Reconstructive Surgery, Gwangmyeong Sungae Hospital, Gwangmyeong, Korea

Arch Plast Surg 2020;47:590-596

https://doi.org/10.5999/aps.2020.01137

In this article, the order of the authors' names was incorrectly stated. The correct order is as follows.

Min Ki Hong, Min Suk Choi, Sung Hoon Koh, Jin Soo Kim, Si Young Roh, Kyung Jin Lee, Dong Chul Lee

We sincerely apologize for the inconvenience that this may have caused. 